\title{
Author Correction: Recovery of nearly 8,000 metagenome-assembled genomes substantially expands the tree of life
}

Donovan H. Parks, Christian Rinke, Maria Chuvochina, Pierre-Alain Chaumeil, Ben J. Woodcroft, Paul N. Evans, Philip Hugenholtz* and Gene W. Tyson*

Correction to: Nature Microbiology https://doi.org/10.1038/s41564-017-0012-7 (2017); published online 11 September 2017.

In the original version of this Article, the authors stated that the archaeal phylum Parvarchaeota was previously represented by only two single-cell genomes (ARMAN-4_5-way FS' and ARMAN-5_5-way FS'). However, these are in fact unpublished, low-quality metagenome-assembled genomes (MAGs) obtained from Richmond Mine, California. In addition, the authors overlooked two higher-quality published Parvarchaeota MAGs from the same habitat, ARMAN-4 (ADCE00000000) and ARMAN-5 (ADHF00000000) (B. J. Baker et al., Proc. Natl Acad. Sci. USA 107, 8806-8811; 2010). The ARMAN-4 and ARMAN-5 MAGs are estimated to be $68.0 \%$ and $76.7 \%$ complete with $3.3 \%$ and $5.6 \%$ contamination, respectively, based on the archaeal-specific marker sets of CheckM. The 11 Parvarchaeota genomes identified in our study were obtained from different Richmond Mine metagenomes, but are highly similar to the ARMAN-4 (ANI of $\sim 99.7 \%$ ) and ARMAN-5 (ANI of $~ 99.6 \%$ ) MAGs. The highest-quality uncultivated bacteria and archaea (UBA) MAGs with similarity to ARMAN-4 and ARMAN-5 are $82.5 \%$ and $83.3 \%$ complete with $0.9 \%$ and $1.9 \%$ contamination, respectively. The Parvarchaeota represents only $0.23 \%$ of the archaeal genome tree and addition of the ARMAN-4 and ARMAN-5 MAGs do not change the conclusions of this Article, but do impact the phylogenetic gain for this phylum. This has now been corrected in all versions of the Article. An updated version of Fig. 5 has also been used to replace the previous version, with the row for Parvarchaeota removed, and Supplementary Table 15 and Supplementary Table 17 have both been replaced to reflect the availability of the two additional Parvarchaeota genomes. In addition, the Methods incorrectly stated that all metagenomes identified as being from studies where MAGs had previously been recovered were excluded from consideration. Metagenomes from studies where MAGs had previously been recovered were retained if the UBA MAGs provided appreciable improvements in genome quality or phylogenetic diversity. All versions of the Article have been updated to indicate the retention of such metagenomes.

Published online: 12 December 2017

https://doi.org/10.1038/s41564-017-0083-5 